\title{
A Decomposition of the Dual Space of Some Banach Function Spaces
}

\author{
Claudia Capone ${ }^{1}$ and Maria Rosaria Formica ${ }^{2}$ \\ ${ }^{1}$ Istituto per le Applicazioni del Calcolo "Mauro Picone", CNR, sez. Napoli, Via P. Castellino 111, \\ 80131 Napoli, Italy \\ 2 Dipartimento di Statistica e Matematica per la Ricerca Economica, \\ Università degli Studi di Napoli "Parthenope", Via Medina 40, 80133 Napoli, Italy
}

Correspondence should be addressed to Maria Rosaria Formica, mara.formica@uniparthenope.it

Received 7 April 2010; Accepted 8 June 2010

Academic Editor: Carlo Sbordone

Copyright (c) 2012 C. Capone and M. R. Formica. This is an open access article distributed under the Creative Commons Attribution License, which permits unrestricted use, distribution, and reproduction in any medium, provided the original work is properly cited.

We give a decomposition for the dual space of some Banach Function Spaces as the Zygmund space $\mathrm{EXP}_{\alpha}$ of the exponential integrable functions, the Marcinkiewicz space $L^{p, \infty}$, and the Grand Lebesgue Space $L^{p), \theta}$.

\section{Introduction}

Let $\Omega$ be a set of Lebesgue measure $|\Omega|<+\infty$.

In this paper, we deal with the following issue. What is the difference between the dual space $X^{*}$ and the associate space $X^{\prime}$ of a Banach Function Space $X$ ?

By associate space $X^{\prime}$ of $X$ we mean the space determined by the associate norm $\rho^{\prime}$ :

$$
\rho^{\prime}(g)=\sup \left\{\int_{\Omega} f g d x: f \in \mathcal{M}^{+}, \rho(f) \leq 1\right\}
$$

as in Definitions 2.3 and 2.4.

If $X$ is a reflexive Banach Function Space, then the dual space $X^{*}$ is canonically isometrically isomorphic to the associate space $X^{\prime}[1$, page 23]. On the other hand, for example, if we consider the Orlicz space $\operatorname{EXP}(\Omega)$ of exponentially integrable functions, which is not reflexive, the associate space $(\operatorname{EXP}(\Omega))^{\prime}$ coincides with the Zygmund space $L \log L(\Omega)$, while the dual can be represented by

$$
(\operatorname{EXP}(\Omega))^{*}=L \log L(\Omega) \oplus(\exp (\Omega))^{\perp},
$$


where $\exp (\Omega)$ is the closure of $L^{\infty}(\Omega)$ with respect to the EXP norm (see [2, Chapter IV], [3] and also Corollary 3.4).

Our aim is to show that the decomposition for the dual space as in (1.2) holds in a more general setting: namely, if $X$ is a rearrangement invariant Banach Function Space on $\Omega$ such that its fundamental function $\varphi_{X}$ verifies

$$
\varphi_{X}(0+)=0,
$$

then,

$$
X^{*}=X^{\prime} \oplus\left(X_{b}\right)^{\perp}
$$

where $X_{b}$ denotes the closure of $L^{\infty}(\Omega)$ in $X$. We stress that, due to assumption (1.3), our argument is much shorter than the corresponding one, treated in Zaanen ([4, Section 70, Theorem 2, page 467]) in the more abstract setting of normed Köethe spaces. (See also [2, Chapter IV, Proposition 2.8 and Theorem 2.11]).

In Section 3, we consider our decomposition in the particular case of $\operatorname{EXP}_{\alpha}$ spaces, Marcinkiewicz spaces, and the Grand Lebesgue Spaces, specifying case by case the expression of the associate space.

Let us note that in general a Banach Function Space $X$ can be identified with a closed subspace of $\left(X^{\prime}\right)^{*}[1]$, while the spaces mentioned in our particular cases verify

$$
X=\left(X^{\prime}\right)^{*}
$$

as shown in Theorem 3.7.

\section{Preliminaries}

Let $\Omega$ be a set of Lebesgue measure $|\Omega|<+\infty$ and let $\mathcal{M}_{o}^{+}$be the set of all measurable functions, whose values lie in $[0,+\infty]$, finite a.e. in $\Omega$.

Definition 2.1. A mapping $\rho: \boldsymbol{M}_{o}^{+} \rightarrow[0,+\infty]$ is called a Banach function norm if, for all $f, g, f_{n}(n=1,2,3, \ldots)$ in $\mathcal{M}_{o}^{+}$, for all constants $a \geq 0$, and for all measurable subsets $E \subset \Omega$, the following properties hold.

$$
\begin{gathered}
\rho(f)=0 \Longleftrightarrow f=0 \text { a.e. in } \Omega, \\
\rho(a f)=a \rho(f), \\
\rho(f+g) \leq \rho(f)+\rho(g), \\
0 \leq g \leq f \text { a.e. in } \Omega \Longrightarrow \rho(g) \leq \rho(f), \\
0 \leq f_{n} \uparrow f \text { a.e. in } \Omega \Longrightarrow \rho\left(f_{n}\right) \uparrow \rho(f), \\
|E|<+\infty \Longrightarrow \rho\left(x_{E}\right)<+\infty, \\
|E|<+\infty \Longrightarrow \int_{E} f d x \leq C_{E} \rho(f)
\end{gathered}
$$

for some constant $C_{E}, 0<C_{E}<\infty$, depending on $E$ and $\rho$, but independent of $f$. 
Definition 2.2. If $\rho$ is a Banach function norm, the Banach space

$$
X=\{f \in \mathcal{M}: \rho(|f|)<+\infty\}
$$

is called a Banach Function Space.

For each $f \in X$, define

$$
\|f\|_{X}=\rho(|f|) .
$$

Recall that the simple functions are contained in every Banach Function Spaces X [1].

Definition 2.3. If $\rho$ is a function norm, its associate norm $\rho^{\prime}$ is defined on $\mathcal{M}_{o}^{+}$by

$$
\rho^{\prime}(g)=\sup \left\{\int_{\Omega} f g d x: f \in \mathcal{M}^{+}, \rho(f) \leq 1\right\} .
$$

Definition 2.4. Let $\rho$ be a function norm and let $X=X(\rho)$ be the Banach Function Space determined by $\rho$. Let $\rho^{\prime}$ be the associate norm of $\rho$. The Banach Function Space $X^{\prime}=X\left(\rho^{\prime}\right)$ determined by $\rho^{\prime}$ is called the associate space of $X$.

In particular, from the definition of $\|f\|_{X}$, it follows that the norm of a function $g$ in the associate space $X^{\prime}$ is given by

$$
\|g\|_{X^{\prime}}=\sup \left\{\int_{\Omega} f g d x: f \in X,\|f\|_{X} \leq 1\right\} .
$$

Definition 2.5. A function $f$ in a Banach Function Space $X$ is said to have absolutely continuous norm in $X$ if $\left\|f X_{E_{n}}\right\|_{X} \rightarrow 0$ for every sequence $\left\{E_{n}\right\}_{n=1}^{\infty}$ satisfying $E_{n} \rightarrow \emptyset$ a.e. The set of all functions in $X$ of absolutely continuous norm is denoted by $X_{a}$. If $X=X_{a}$, then the space $X$ itself is said to have absolutely continuous norm.

Definition 2.6. Let $f \in \mathcal{M}_{\mathrm{o}}$. The function

$$
\mu_{f}(\lambda)=|\{x \in \Omega:|f(x)|>\lambda\}| \quad \forall \lambda \geq 0
$$

is called the distribution function of $f$. The decreasing rearrangement of $f, f^{*}$, is defined on $[0,|\Omega|]$ by

$$
f^{*}(t)=\inf \left\{\lambda>0: \mu_{f}(\lambda) \leq t\right\},
$$

where here we use the convention $\inf \emptyset=+\infty$.

Two functions having the same distribution function are called equimeasurable.

Let us recall that a function norm $\rho$ is said to be rearrangement invariant (briefly, "r.i.") if $\rho(f)=\rho(g)$ for every couple of equimeasurable functions. The Banach Function Space arising from a r.i. function norm is called a rearrangement-invariant space. 
By $f^{* *}:(0, \infty) \rightarrow[0, \infty]$, we denote the function given by

$$
f^{* *}(t)=\frac{1}{t} \int_{0}^{t} f^{*}(s) d s, \quad(t>0) .
$$

The function $f^{* *}$ is nonincreasing and verifies $f^{*}(t) \leq f^{* *}(t) \quad(t>0)$.

Definition 2.7. Let $X$ be a r.i. Banach Function Space determined by a function norm $\rho$. For each $t \in[0,|\Omega|]$, let $E_{t} \subseteq \Omega$ be a set of measure $t$. The fundamental function of $X, \varphi_{X}(t)$, is defined by

$$
\varphi_{X}(t)=\rho\left(X_{E_{t}}\right)=\left\|X_{E_{t}}\right\|_{X} \cdot
$$

Definition 2.8. Let $1 \leq p \leq \infty$ and $\alpha \in \mathbf{R}$, then the Zygmund space $L^{p}(\log L)^{\alpha}(\Omega)$ is the set of all measurable functions $f$ in $\Omega$ for which the quantity

$$
\|f\|_{L^{p}(\log L)^{\alpha}(\Omega)}=\left\|\left(1+\log \left(\frac{|\Omega|}{t}\right)\right)^{\alpha} f^{*}(t)\right\|_{L^{p}(0,|\Omega|)}
$$

is finite.

For $p=1$ and $\alpha=1$ we will replace $L^{1}(\log L)^{1}(\Omega)$ by $L(\log L)(\Omega)$.

With these notations, the usual space $\operatorname{EXP}_{\alpha}$ of the exponentially integrable functions corresponds to the Zygmund space $\left(L^{\infty} /(\log L)^{1 / \alpha}\right)(\Omega)$ and consists of all measurable functions $f$ in $\Omega$ for which the quantity

$$
\|f\|_{L^{\infty} /(\log L)^{1 / \alpha}(\Omega)}=\|f\|_{\operatorname{EXP}_{\alpha}(\Omega)}=\sup _{0<t<|\Omega|}\left(1+\log \left(\frac{|\Omega|}{t}\right)\right)^{-1 / \alpha} f^{*}(t)
$$

is finite.

All these spaces are particular cases of the Orlicz spaces.

Let $\phi:[0, \infty) \rightarrow[0, \infty)$ be a right-continuous, increasing function, such that $\phi(0)=0$ and $\lim _{t \rightarrow \infty} \phi(t)=\infty$, then the function defined by

$$
\Phi(t)=\int_{0}^{t} \phi(s) d s
$$

is called $N$ function; it is a continuous, convex, increasing function such that $\lim _{t \rightarrow \infty}(\Phi(t) / t)=$ $+\infty$ and $\lim _{t \rightarrow 0}(\Phi(t) / t)=0$.

Definition 2.9. The Orlicz space $L^{\Phi}(\Omega)$ consists of all measurable functions $f$ on $\Omega$ for which there exists some $\lambda>0$ such that

$$
f_{\Omega} \Phi\left(\frac{|f|}{\lambda}\right)<\infty
$$

where $f_{\Omega}$ stands for $(1 /|\Omega|) \int_{\Omega}$. 
This is a Banach space with respect to the Luxemburg norm:

$$
\|f\|_{L^{\Phi}}(\Omega)=\inf \left\{\lambda>0: f_{\Omega} \Phi\left(\frac{|f(x)|}{\lambda}\right) d x \leq 1\right\} .
$$

The Orlicz spaces are a standard example of rearrangement-invariant Banach Function Space: the associate space of $L^{\Phi}(\Omega)$ is given by $L^{\widetilde{\Phi}}(\Omega)$, where $\widetilde{\Phi}$ denotes the complementary function of $\Phi$, defined by

$$
\widetilde{\Phi}(t)=\max \{s t-\Phi(s): s \geq 0\} .
$$

Moreover, we notice that, for $\Phi(t)=t^{p}, \Phi(t)=t^{p}(\log t)^{\alpha}$, and $\Phi(t)=e^{t^{\alpha}}-1$, the Orlicz space associated reduces, respectively, to the spaces $L^{p}(\Omega), L^{p}(\log L)^{\alpha}(\Omega)$ and to $\operatorname{EXP}_{\alpha}(\Omega)$.

Definition 2.10. Given $1 \leq p, q \leq \infty$, the Lorentz space $L^{p, q}(\Omega)$ consists of all measurable functions $f$ in $\Omega$ for which

$$
\|f\|_{p, q}=\left\{\begin{array}{lc}
\int_{0}^{\infty}\left[t^{1 / p} f^{*}(t)\right]^{q} \frac{d t}{t}, & 0<q<\infty, \\
\sup _{t>0} t^{1 / p} f^{*}(t), & q=\infty
\end{array}\right.
$$

is finite.

The space $L^{p, \infty}(\Omega)=$ Weak- $L^{p}(\Omega)$ is known as the Marcinkiewicz space, and it is another example of r.i. Banach Function Space.

The quantity (2.16) is not a norm since the triangle inequality may fail; however, for $p>1$, replacing $f^{*}(t)$ with $f^{* *}(t)$, we obtain a norm equivalent to (2.16). to

In particular, for $q=\infty$, in the case of a nonatomic measure space, (2.16) is equivalent

$$
\sup \left\{|E|^{1 / p-1} \int_{E}|f| d x, E \subset \Omega \text { measurable }\right\}
$$

Now, we recall the definitions of Grand and Small Lebesgue Spaces, introduced, respectively, in [5] and in [6].

Definition 2.11. Let $1<p<+\infty$ and $\theta \geq 0$; the Grand Lebesgue Space $L^{p), \theta}$ is the Banach Function Space of all measurable functions $f$ on $\Omega$ such that

$$
\|f\|_{p), \theta}=\sup _{0<\varepsilon<p-1}\left(\varepsilon^{\theta} f_{\Omega}|f|^{p-\varepsilon} d x\right)^{1 /(p-\varepsilon)}
$$

is finite.

Notice that

$$
L^{p), 0}(\Omega)=L^{p}(\Omega), \quad L^{p), 1}(\Omega)=L^{p)}(\Omega)
$$


If $1<p<+\infty$ and $p^{\prime}$ is its Hölder conjugate exponent, according to [7], the Small Lebesgue Space $L^{\left(p^{\prime}, \theta\right.}$ can be identified as the set of all measurable functions $f$ on $\Omega$ such that

$$
\|f\|_{\left(p^{\prime}, \theta\right.}=\sup \left\{\left|\int_{\Omega} f g d x\right|:\|f\|_{L^{p, \theta}(\Omega)} \leq 1\right\}
$$

is finite.

The Grand and Small Lebesgue Spaces are r.i. Banach Function Spaces [7].

Definition 2.12. A vector space $V$ is the direct sum of its subspaces $U$ and $W$, denoted by $V=$ $U \oplus W$, if and only if

$$
\begin{gathered}
V=U+W=\{u+w: u \in U, w \in W\}, \\
V \cap W=\{0\} .
\end{gathered}
$$

Elements $v$ of the direct sum $U \oplus W$ are representable uniquely in the form

$$
u+w: u \in U, \quad w \in W
$$

Definition 2.13. Let $X$ be a Banach space and $M \subset X$ a vectorial subspace of $X$. The orthogonal space $M^{\perp}$ of $M$ is

$$
M^{\perp}=\left\{f \in X^{*}:\langle f, x\rangle=0, \quad \forall x \in M\right\},
$$

where $\langle.,$.$\rangle is the duality inner product.$

It is known that $M^{\perp}$ is a closed subspace of $X^{*}$.

We conclude this section by recalling some classical results, which will be useful in the sequel.

Theorem 2.14 (Hölder's inequality [1]). Let X be a Banach Function Space with associate space $X^{\prime}$. If $f \in X$ and $g \in X^{\prime}$, then $f g$ is integrable and

$$
\int_{\Omega} f g d x \leq\|f\|_{X}\|g\|_{X^{\prime}}
$$

Lemma 2.15 (see [1, Lemma 2.6, page 10]). In order that a measurable function $g$ belongs to the associate space $X^{\prime}$, it is necessary and sufficient that $f g$ is integrable for every $f$ in $X$.

Theorem 2.16 (see [1, Theorem 2.7, page 10]). Every Banach Function Space X coincides with its second associate space $X^{\prime \prime}=\left(X^{\prime}\right)^{\prime}$.

Theorem 2.17 (see [1, Theorem 2.9, page 13]). The associate space $X^{\prime}$ of a Banach Function Space $X$ is canonically isometrically isomorphic to a closed norm-fundamental subspace of the Banach space dual $X^{*}$ of $X$.

Proposition 2.18 (see [1, Proposition 2.10, page 13]). If $X$ and $Y$ are Banach Function Spaces and $X \subset Y$ (continuous embedding), then $Y^{\prime} \subset X^{\prime}$ (continuous embedding). 
Theorem 2.19 (see [1, Theorem 3.11, page 18]). Let X be a Banach Function Space. Then, $X_{a} \subseteq$ $X_{b} \subseteq X$.

Corollary 2.20. If $X_{a}=X$, then $X_{b}=X$.

Theorem 2.21 (see [1, Theorem 3.13, page 19]). The subspaces $X_{a}$ and $X_{b}$ coincide if and only if the characteristic function $X_{E}$ has absolutely continuous norm for every set $E$ of finite measure.

Theorem 2.22 (see [1, Corollary 4.2, page 23]). Let X be a Banach Function Space. If $X_{a}$ contains the simple functions, then $\left(X_{a}\right)^{*}=X^{\prime}$.

Theorem 2.23 (see [1, Corollary 4.3, page 23]). The Banach space dual X* of a Banach Function Space $X$ is canonically isometrically isomorphic to the associate space $X^{\prime}$ if and only if $X$ has absolutely continuous norm.

Theorem 2.24 (see [1, Theorem 5.5, page 67]). Let $(\Omega, \mu)$ be a totally $\sigma$-finite nonatomic measure space and let $X$ be an arbitrary rearrangement-invariant space over $(\Omega, \mu)$. The following conditions on $X$ are equivalent:

(i) $\lim _{t \rightarrow 0^{+}} \varphi_{X}(t)=0$;

(ii) $X_{a}=X_{b}$;

(iii) $\left(X_{b}\right)^{*}=X^{\prime}$,

where $\varphi_{X}(t)$ is the fundamental function of $X$.

\section{Main Results}

In this Section, we establish a decomposition for the dual space of a r.i. Banach Function Space.

Theorem 3.1. Let $X$ be a rearrangement-invariant Banach Function Space on $\Omega$. For each $t \in$ $[0,|\Omega|]$, let $E$ be a subset of $\Omega$ with $|E|=t$ and let $\varphi_{X}(t)$ be the fundamental function of $X$.

If

$$
\lim _{t \rightarrow 0^{+}} \varphi_{X}(t)=0
$$

then the following decomposition

$$
X^{*}=X^{\prime} \oplus\left(X_{b}\right)^{\perp}
$$

holds.

Proof. Let $l \in X^{*}$, for all measurable sets $F$ in $\Omega$, we define the set function

$$
v(F)=l\left(X_{F}\right),
$$


which is $\sigma$-additive and absolutely continuous with respect to the Lebesgue measure $|F|$. Thus, $v$ has a locally integrable Radon-Nikodym derivative $g$ and

$$
l(f)=\int_{\Omega} f g d x, \quad \text { for any } f \in L^{\infty}(\Omega) .
$$

Since $l \in X^{*}$ for all $f \in X$, it is

$$
l(f) \leq K\|f\|_{X}
$$

where $K$ is a constant. Hence, for all $f \in L^{\infty}$,

$$
\int_{\Omega} f g d x \leq K\|f\|_{X}
$$

By Lemma 2.15, it follows that $g \in X^{\prime}$.

To any $g \in X^{\prime}$, we can associate the functional

$$
l_{g}: f \in X_{b} \longrightarrow \int_{\Omega} f g d x
$$

By Hölder's inequality, $l_{g}$ belongs to $X_{b}^{*}$, which is equivalent to $X^{\prime}$ thanks to Theorem 2.24 .

Finally, let $l_{s}$ be defined by $l_{s}=l-l_{g}$, then $l_{s}(f)=\left\langle l_{s}, f\right\rangle=0$ for all $f \in X_{b}$. Therefore, $l_{s}$ belongs to $\left(X_{b}\right)^{\perp}$.

Hence,

$$
l=l_{g}+l_{s} \in X^{\prime}+X_{b}^{\perp}
$$

Since it is easily seen that $X^{\prime}$ and $X_{b}^{\perp}$, subspaces of $X^{*}$, verify $X^{\prime} \cap X_{b}^{\perp}=\{0\}$, then the proof is complete.

Remark 3.2. Let us point out that, by Theorem 2.24, the decomposition (3.2) can also be written as

$$
\begin{aligned}
& X^{*}=\left(X_{b}\right)^{*} \oplus\left(X_{b}\right)^{\perp}, \\
& X^{*}=\left(X_{a}\right)^{*} \oplus\left(X_{a}\right)^{\perp} .
\end{aligned}
$$

Corollary 3.3. Let X be an Orlicz space, then

$$
\begin{aligned}
X^{*} & =X^{\prime} \oplus\left(X_{b}\right)^{\perp} \\
& =\left(X_{b}\right)^{*} \oplus\left(X_{b}\right)^{\perp} \\
& =\left(X_{a}\right)^{*} \oplus\left(X_{a}\right)^{\perp} .
\end{aligned}
$$


Proof. If $X=L^{\Phi}(\Omega)$ is an Orlicz space, then the fundamental function is

$$
\left.\left.\varphi_{X}(t)=\frac{1}{\Phi^{-1}(1 / t)}, \quad \forall t \in\right] 0,|\Omega|\right]
$$

(see [7]). Therefore, $\lim _{t \rightarrow 0^{+}} \varphi_{X}(t)=0$ and the claim follows from Theorem 3.1 and Remark 3.2.

Corollary 3.4. Let $X=\operatorname{EXP}_{\alpha}(\Omega), \alpha>0$, then

$$
\begin{aligned}
\left(\operatorname{EXP}_{\alpha}(\Omega)\right)^{*} & =L \log ^{1 / \alpha} L(\Omega) \oplus\left(\exp _{\alpha}(\Omega)\right)^{\perp} \\
& =\left(\exp _{\alpha}(\Omega)\right)^{*} \oplus\left(\exp _{\alpha}(\Omega)\right)^{\perp},
\end{aligned}
$$

where $\exp _{\alpha}(\Omega)$ denotes the closure of $L^{\infty}(\Omega)$ in $\operatorname{EXP}_{\alpha}(\Omega)$.

Proof. The result follows by Corollary 3.3, and by $\left(\operatorname{EXP}_{\alpha}(\Omega)\right)^{\prime}=L \log ^{1 / \alpha} L(\Omega), \alpha>0$, (see $[1])$.

Corollary 3.5. Let $p \in] 1, \infty\left[, p^{\prime}\right.$ be its Hölder conjugate exponent and $X=L^{p, \infty}(\Omega)$, then

$$
\left(L^{p, \infty}(\Omega)\right)^{*}=L^{p^{\prime}, 1}(\Omega) \oplus\left(L_{b}^{p, \infty}(\Omega)\right)^{\perp} .
$$

Proof. The Marcinkievicz space $L^{p, \infty}(\Omega)$ is the largest of all rearrangement-invariant spaces having the same fundamental function as $L^{p}(\Omega)$ (see [1]), which is

$$
\varphi_{L^{p}}(t)=t^{1 / p}
$$

Moreover, the associate space of $L^{p, \infty}(\Omega)$ (see [1]) is, up to equivalence of norms, the Lorentz space $L^{p^{\prime}, 1}(\Omega)$.

Therefore, the statement easily follows by Theorem 3.1.

A decomposition of the dual of $L^{p, \infty}$ was also given in [8].

Corollary 3.6. Let $p \in] 1, \infty\left[, \theta \geq 0\right.$ and $X=L^{p), \theta}(\Omega)$, then

$$
\left(L^{p), \theta}(\Omega)\right)^{*}=L^{\left(p^{\prime}, \theta\right.}(\Omega) \oplus\left(L_{b}^{p), \theta}(\Omega)\right)^{\perp}
$$

Proof. Let $\varphi_{X}(t)$ be the fundamental function of the space $L^{p), \theta}(\Omega)$, then

$$
\varphi_{X}(t) \approx t^{1 / p}\left[\log \left(\frac{1}{t}\right)\right]^{-\theta / p}
$$

as $t \rightarrow 0^{+}$(see [7]).

Therefore the claim easily follows by Theorem 3.1 and by the relation $\left(L^{p), \theta}(\Omega)\right)^{\prime}=$ $L^{\left(p^{\prime}, \theta\right.}(\Omega)$ (see [7]). 
In the next theorem, we show the relation between a Banach Function Space $X$ and the dual of its associate space $\left(X^{\prime}\right)^{*}$.

Theorem 3.7. Let X be a Banach Function Space, then the following inclusion

$$
X \subseteq\left(X^{\prime}\right)^{*}
$$

holds, with equality occurring if and only if the associate space $X^{\prime}$ of $X$ has absolutely continuous norm.

Proof. By Theorem 2.17 applied to the Banach Function Space $X^{\prime}$, we may identify $\left(X^{\prime}\right)^{\prime}$ with a closed subspace of $\left(X^{\prime}\right)^{*}$; hence, Theorem 2.16 implies

$$
X=X^{\prime \prime}=\left(X^{\prime}\right)^{\prime} \subseteq\left(X^{\prime}\right)^{*}
$$

Furthermore, if $X^{\prime}$ has absolutely continuous norm, that is $X^{\prime}=X_{a}^{\prime}$, since every Banach Function Space contains the simple functions, by Theorem 2.22 applied to the space $X^{\prime}$ and by Theorem 2.16, we have $\left(X^{\prime}\right)^{*}=\left(X_{a}^{\prime}\right)^{*}=\left(X^{\prime}\right)^{\prime}=X^{\prime \prime}=X$.

On the other hand, if $X=\left(X^{\prime}\right)^{*}$, then $\left(X^{\prime}\right)^{\prime}=X=\left(X^{\prime}\right)^{*}$, and Theorem 2.23 yields that $X^{\prime}$ has absolutely continuous norm.

Remark 3.8. An example of a Banach Function Space verifying the proper inclusion in (3.18) is given by the Lebesgue space $L^{1}$. In fact, if $X=L^{1}$, then

$$
\left(X^{\prime}\right)^{*}=\left(\left(L^{1}\right)^{\prime}\right)^{*}=\left(L^{\infty}\right)^{*} \supset L^{1}
$$

as confirmed by the fact that $L^{\infty}$ has not absolutely continuous norm.

\section{References}

[1] C. Bennett and R. Sharpley, Interpolation of Operators, Academic Press, 1988.

[2] M. M. Rao and Z. D. Ren, Theory of Orlicz Spaces, Pure and Applied Mathematics, vol. 146, Marcel Dekker, New York, NY, USA, 1991.

[3] M. Carozza and C. Sbordone, "The distance to $L^{\infty}$ in some function spaces and applications," Differential and Integral Equations, vol. 10, no. 4, pp. 599-607, 1997.

[4] A. C. Zaanen, Integration, Completely Revised Edition of An Introduction to the Theory of Integration, North-Holland Publishing, Amsterdam, The Netherlands, 1967.

[5] T. Iwaniec and C. Sbordone, "On the integrability of the Jacobian under minimal hypotheses," Archive for Rational Mechanics and Analysis, vol. 119, no. 2, pp. 129-143, 1992.

[6] A. Fiorenza, "Duality and reflexivity in grand Lebesgue spaces," Collectanea Mathematica, vol. 51, no. 2, pp. 131-148, 2000.

[7] C. Capone and A. Fiorenza, "On small Lebesgue spaces," Journal of Function Spaces and Applications, vol. 3, no. 1, pp. 73-89, 2005.

[8] M. Cwikel, "The dual of Weak $L^{p}$," Annales de l'Institut Fourier, vol. 25, no. 2, pp. 81-126, 1975. 


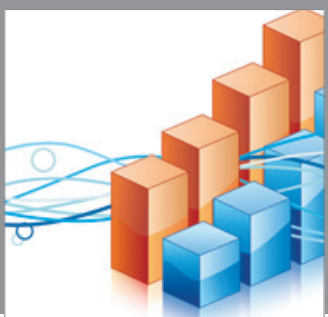

Advances in

Operations Research

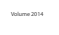

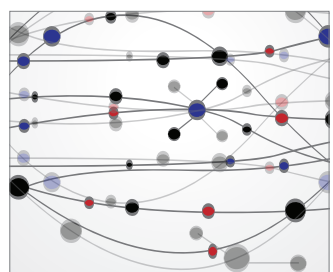

\section{The Scientific} World Journal
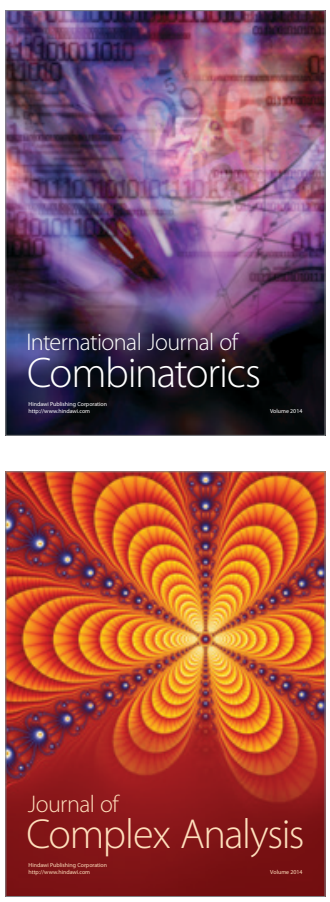

International Journal of

Mathematics and

Mathematical

Sciences
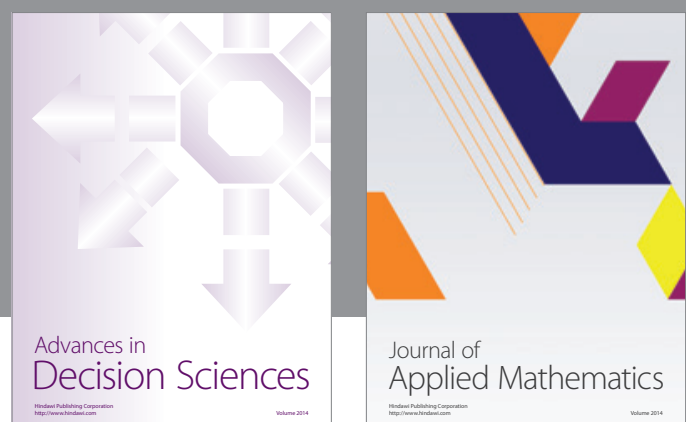

Journal of

Applied Mathematics
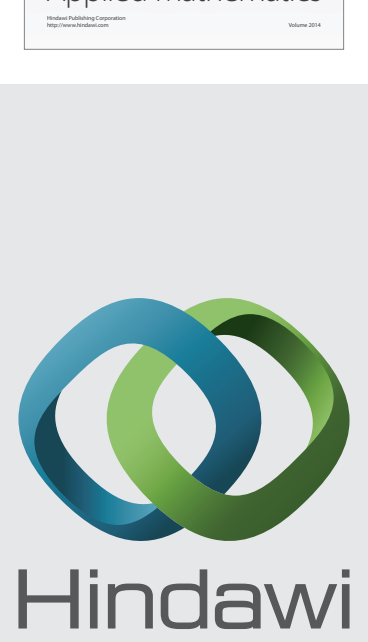

Submit your manuscripts at http://www.hindawi.com
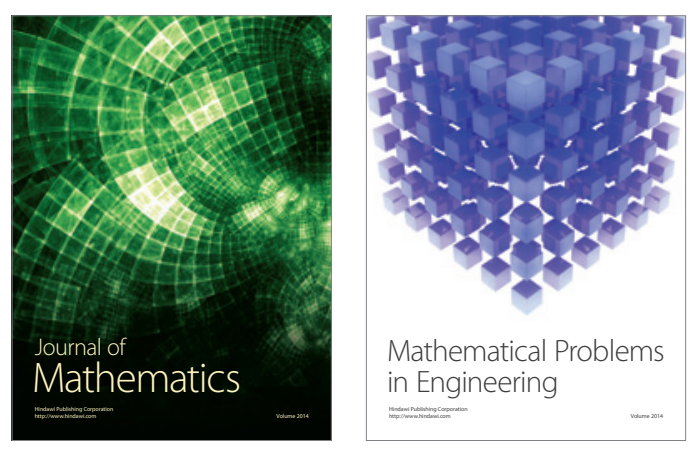

Mathematical Problems in Engineering
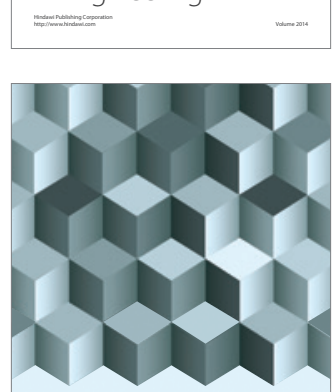

Journal of

Function Spaces
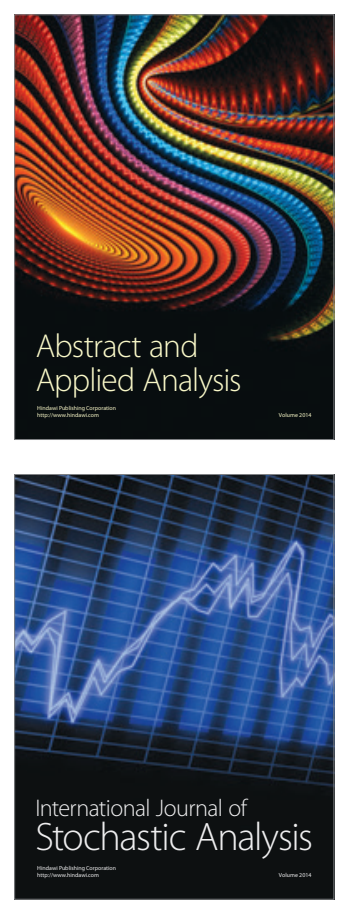

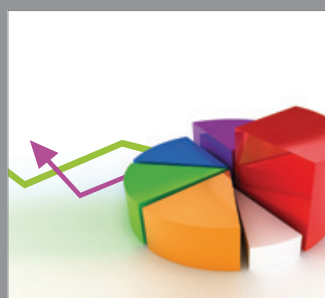

ournal of

Probability and Statistics

Promensencen
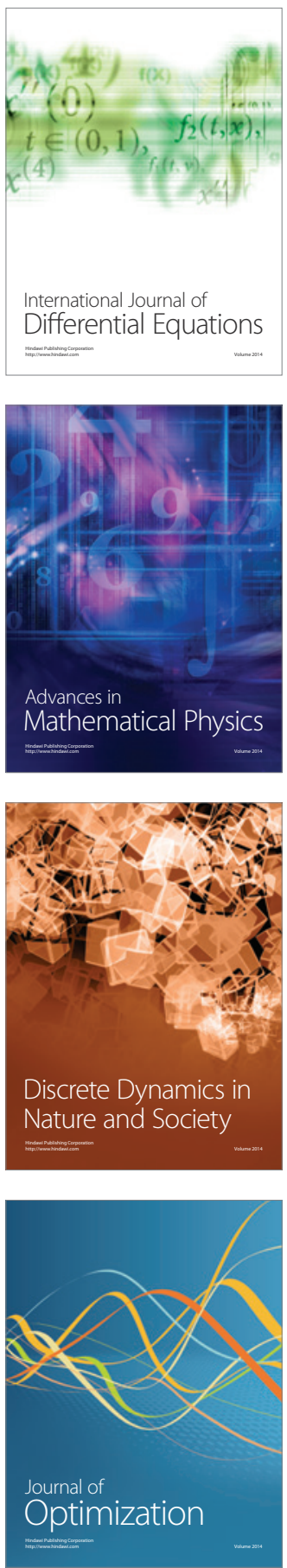